\title{
Programa informativo sobre Deficiência mental E INClusão: MUDANÇAS NAS ATITUDES SOCIAS DE CRIANÇAS SEM DEFICIÊNCIA ${ }^{\mathbf{1}}$
} INFORMATIVE PROGRAM ABOUT MENTAL RETARDATION AND INCLUSION: ADJUSTMENT IN SOCIAL ATTITUDES OF CHILDREN WITHOUT DISABILITIES

\author{
Camila Mugnai VIEIRA ${ }^{2}$ \\ Fátima Elisabeth DENARI ${ }^{3}$
}

\begin{abstract}
RESUMO: a educação inclusiva depende de um ambiente escolar acolhedor e adequado às necessidades de todos. Assim, as investigações das atitudes sociais na escola e das intervenções para modificá-las são fundamentais. Os objetivos desta pesquisa foram analisar concepções, sentimentos e atitudes de crianças não-deficientes sobre a deficiência mental e a inclusão e avaliar os efeitos de um programa informativo que trata da temática. Participaram do estudo quarenta crianças de duas salas de primeira série de uma escola estadual de Marília-SP. Uma das salas participou como grupo controle. Todas as crianças passaram por um pré e um pós-teste, na forma de entrevistas individuais sobre o tema e de aplicação de uma escala infantil de atitudes sociais em relação à inclusão. O grupo experimental participou do programa informativo, composto por treze encontros semanais, nos quais foram discutidas as limitações e as possibilidades das pessoas com deficiência mental, o atendimento especializado, sua escolarização e aspectos familiares e sociais, utilizando estratégias lúdicas e educacionais variadas. Os dados coletados nas entrevistas foram categorizados e analisados em seu conteúdo. Com a escala, foram obtidos escores individuais. Foram realizados cálculos estatísticos para verificar a significância das diferenças entre os grupos. Neste trabalho são discutidos os dados obtidos com a escala e o cruzamento destes com dados das entrevistas. Os resultados das entrevistas e da escala indicaram diversas mudanças nas atitudes das crianças em relação à inclusão, porém as relações entre vários destes dados não puderam ser afirmadas estatisticamente. Tendo em vista esses resultados, permanece a necessidade de ampliação das pesquisas sobre as relações entre os fenômenos apresentados.
\end{abstract}

PALAVRAS-CHAVE: Educação Especial. Inclusão Educacional. Atitudes Sociais. Programas.

\begin{abstract}
This article presents part of a study that analyzed the concepts, feelings and attitudes of children without disabilities about mental retardation and inclusion and evaluated the effects of an informative program that deals with the issue. The study included forty children from two first grade classrooms in a public school in Marília-SP. One classroom participated as a control group. All children underwent pre and post tests in the form of interviews on the subject and a scale of children's social attitudes towards inclusion was applied. The experimental group participated in the informative program, composed of thirteen weekly meetings, in which the limitations and possibilities of people with mental retardation, specialized care, their schooling and family and social aspects, were discussed, using various educational and recreational strategies. The data collected in the interviews were categorized and content analysis was conducted. With the scale, individual scores were obtained. Statistical calculations were performed to verify the significance of differences between groups. In this paper we discuss the data obtained with the scale which were crossed with interview data. The results of the interviews and the scale indicated several changes in children's attitudes towards inclusion, but relations between many of these data could not be statistically confirmed. These results indicate the importance of expanding the research on the relationship between the phenomena presented.
\end{abstract}

KEYWORDS: Special Education. Inclusion. Social Attitudes. Programs.

${ }^{1}$ Pesquisa financiada pela CAPES - Coordenação de Aperfeiçoamento de Pessoal de Nível Superior - na forma de Bolsa de Mestrado PROESP.

${ }^{2}$ Psicóloga formada pela Universidade Estadual de Londrina, Mestre em Educação Especial pelo Programa de PósGraduação em Educação Especial da Universidade Federal de São Carlos. Doutoranda do programa de Pós-graduação em Educação da Unesp - Marília. Docente da Faculdade de Medicina de Marília - Famema. camilamugnai@gmail.com

${ }^{3}$ Pedagoga, mestre em Educação Especial pelo Programa de Pós Graduação em Educação Especial (PPGEES) da Universidade Federal de São Carlos; doutora em Educação pelo Programa de Pós Graduação em Educação da Universidade Federal de São Carlos, docente do PPGEES. fadenari@terra.com.br 


\section{INTRODUÇÃo}

Diversas discussões referentes à inclusão vêm sendo levantadas, sobre os aspectos políticos, educacionais e sociais que envolvem sua constituição, entendimentos e práticas. $O$ fato é que para se efetivar uma real inclusão educacional e social, são necessárias muitas transformações, reestruturação das escolas em diferentes níveis, tais quais curriculares, estruturais, ideológicos, de formação e capacitação profissional, além de reflexões de questões econômicas e políticas envolvidas (STAINBACK; STAINBACK, 1999).

Além disso, a inclusão não pode ser vista apenas como um desafio de políticas educacionais ou de transformações pedagógicas e curriculares. É preciso que se compreenda que a inclusão é um processo subjetivo e inter-relacional. Trata-se de pessoas em relação, pessoas com crenças, valores e atitudes construídas socialmente ao longo da história de sua cultura e de sua história individual.

Assim, é fundamental a ampliação de estudos que não se ocupem apenas, das pessoas com necessidades educacionais especiais ou com deficiência, mas também, das relações destes com o mundo à sua volta, das reações que as pessoas sem deficiência têm diante destas, das concepções e das representações construídas na sociedade acerca dos deficientes. Isto afeta diretamente o tratamento recebido por profissionais, familiares e sociedade em geral nas relações institucionais e cotidianas, além de refletirem na qualidade de vida de todos envolvidos, nos diferentes contextos.

Omote (1994) ressalta a importância de se buscar compreender como os sujeitos interpretam as deficiências, organizam suas percepções em relação a elas e constroem suas relações interpessoais com as pessoas deficientes. De acordo este autor, estudos nesse sentido, que se preocupam com as relações sociais e a diversidade humana, podem ampliar a compreensão sobre pessoas deficientes e não-deficientes. Por conseguinte, a compreensão de tais interações pode levar ao desenvolvimento de estratégias que facilitem o estabelecimento de relações mais justas para todos, a partir do respeito à diversidade.

As concepções ou representações que as pessoas constroem sobre o mundo, sobre as outras pessoas e sobre si mesmas estão ligadas a aspectos históricos, sociais, cognitivos, afetivos e comportamentais. Logo, as concepções são construídas de acordo com o contexto sócio-histórico e educacional, abrangendo, também, aspectos individuais, como experiências pessoais, sentimentos e crenças. São construídas em uma relação complexa, entre a subjetividade e a objetividade, o singular e o plural (DENARI, 2006). Assim como são construídas nessa relação entre os indivíduos e o mundo, as concepções ou entendimentos sobre a realidade afetam as atitudes interpessoais.

As atitudes são predisposições psíquicas ou afetivas, podendo ser favoráveis ou desfavoráveis (positivas ou negativas) com relação a uma pessoa, grupo, objetos ou fenômenos, situações e idéias. As atitudes preparam, em princípio, uma ação (AMARAL, 1992). No entanto, há autores que defendem 
que as mudanças de comportamentos, por exemplo, podem alterar as atitudes, como Bem, ainda em 1973. De todo modo, entende-se que as atitudes estão relacionadas a emoções, cognições, comportamentos e relações sociais, no sentido de influenciarem e serem influenciadas por estes.

Segundo Omote (2005), o conceito de atitudes sociais parece adequar-se ao estudo das reações das pessoas em relaçãoà inclusão devidoà atualidadeerelevância do tema. Além disso, as reações expressas diante da inclusão envolvem valores pessoais sobre direitos, normas sociais e padrões de normalidade e certamente, possuem importantes elementos cognitivos, emocionais e comportamentais, que são os três componentes das atitudes sociais, segundo vários autores.

Assim, pensando em contribuir com estes entendimentos e reflexões, este artigo propõe um recorte de uma Dissertação de Mestrado de Vieira (2006) que teve como objetivos analisar concepções, sentimentos e atitudes de crianças não-deficientes sobre a deficiência mental ${ }^{4}$ e a inclusão e avaliar os efeitos de um programa informativo que trata da temática.

Nesteartigo, são apresentados alguns dos resultados da pesquisa referentes à aplicação de uma escala infantil de atitudes sociais em relação à inclusão e do cruzamento destes resultados com alguns dados obtidos em entrevistas com as crianças, ressaltando os efeitos da intervenção sobre as atitudes das crianças em relação à inclusão.

\section{Método}

Participaram do estudo 40 crianças não-deficientes de duas salas de primeira série (20 de cada sala), de uma escola estadual de uma cidade de médio porte do noroeste paulista, que atendia alunos com deficiência mental em classes especiais, salas de recursos e salas comuns. Os alunos de uma das salas formaram o "grupo experimental" (GE) e, os da outra sala participaram como "grupo controle" (GC).

Os seguintes instrumentos foram utilizados para a coleta de dados:

1. Roteiro semiestruturado para entrevistas com as crianças: composto por 10 questões abertas, algumas com desdobramentos. Esse roteiro foi elaborado com base em instrumentos de estudos anteriores (BALEOTTI; OMOTE, 2003; FERREIRA, 1998; MARTINS, 1999) e passou por um estudo preliminar, antes de sua aplicação.

2. Escala infantil de atitudes sociais em relação à inclusão: composta por 27 itens que apresentam afirmações sobre a inclusão e três alternativas de respostas (sim, não e não sei). Essa escala foi elaborada e validada a partir de estudos realizados pelo Grupo de Pesquisa Diferença, Desvio e Estigma, da UNESP de Marília-SP (BALEOTTI; OMOTE, 2003) e tem como objetivo investigar as

\footnotetext{
4 Sabe-se que a terminologia mais utilizada atualmente é "deficiência intelectual". Apesar disso, optou-se pelo termo "deficiência mental" para manter a terminologia utilizada na dissertação que originou o presente trabalho.
} 
atitudes sociais de crianças com relação à inclusão de deficientes. A escala foi utilizada no pré e no pós-teste.

Todas as crianças passaram por um pré e um pós-teste, na forma de entrevistas individuais, sobre o tema e aplicação da escala de atitudes.

O pré-teste iniciou-se por uma contextualização da temática para as crianças por meio de um vídeo que apresentava crianças com deficiência em seu cotidiano escolar (FERREIRA, 1998). Após este, cada criança desenhou, ela própria e uma criança deficiente mental (como ela conhecia ou imaginava que fosse). A partir do desenho e do roteiro de entrevistas, as crianças foram entrevistadas individualmente com objetivo de investigar o contato anterior e atual da criança com deficientes, o acesso a informações sobre o assunto e suas concepções, atitudes e sentimentos com relação à deficiência mental e à inclusão. As entrevistas tiveram duração de cinco a vinte minutos, aproximadamente, e foram gravadas.

Após as entrevistas, foi aplicada a Escala infantil de atitudes sociais em relação à inclusão (BALEOTTI; OMOTE, 2003). A aplicação da escala foi coletiva e monitorada - a pesquisadora leu cada item para as crianças, fazendo as explicações necessárias de seus conteúdos e esperando que todas tivessem respondido um item para iniciar o seguinte. As turmas foram divididas em dois grupos para que a aplicação pudesse ser melhor supervisionada. Assim, aproximadamente a metade das crianças passou pela aplicação da escala e, logo em seguida, as crianças restantes passaram pelo mesmo procedimento. A aplicação foi feita na sala de vídeo da escola.

O grupo experimental participou do programa informativo, baseado no programa de Ferreira (1998), descrito no livro Aprendendo sobre a deficiência mental: um programa para crianças. O programa foi composto por treze encontros semanais de aproximadamente uma hora de duração. Entre os conteúdos trabalhados no programa estão:

- A contextualização da deficiência mental na diversidade humana;

- A explicação da deficiência mental, de acordo com as possibilidades de compreensão das crianças;

- A visão do deficiente mental como capaz, com habilidades, com necessidades especiais e limitações, mas podendo exercer sua cidadania e ter uma vida plena, com relacionamentos afetivos e produtividade;

- Conteúdos relacionados à necessidade de intervenção especializada precoce, relacionamentos familiares e escolarização.

Os encontros aconteceram na própria escola, no horário de aula das crianças e com a participação da professora da sala. Para os encontros, foram preparadas atividades lúdicas e educativas variadas e materiais diversos, como fantoches, revistas, livros infantis, roupas e objetos para teatro, entre outros. 
Após o desenvolvimento do programa, todas as crianças passaram pelo pós-teste, no qual adotou-se o mesmo procedimento do pré-teste.

As entrevistas com as crianças foram transcritas na íntegra. A pesquisadora realizou várias leituras das entrevistas e as respostas foram organizadas em categorias, a partir da análise de conteúdo. Após isso, o material foi encaminhado a dois juízes para a codificação das respostas, segundo as categorias propostas pela pesquisadora, mas independentemente da organização feita por ela e independentemente um do outro. O maior índice de concordância obtido foi de $87,3 \%$.

Alguns dados obtidos nas entrevistas passaram por uma análise estatística para a verificação da significância das diferenças entre os grupos. Aplicou-se a Prova de Fisher, comparando-se o pré-teste do grupo experimental com o pré-teste do grupo controle. Todos os resultados indicaram não haver diferença significativa entre os grupos no pré-teste. Posteriormente, aplicou-se a Prova de Fisher para comparar o pós-teste do grupo experimental com o pós-teste do grupo controle, visando verificar se as diferenças apresentadas pelos grupos no pós-teste eram significativas.

Para a análise dos dados da escala infantil, foram atribuídos escores aos itens da mesma. Cada item da escala foi pontuado com os valores convencionados $-1,0$ ou 1 . O valor -1 foi atribuído quando o respondente discordou do enunciado favorável à inclusão ou concordou com o enunciado desfavorável à inclusão; o valor 1 foi atribuído quando o respondente concordou com o enunciado favorável à inclusão ou discordou do enunciado desfavorável à inclusão; e o valor 0 foi atribuído quando o respondente assinalou que não sabia opinar a respeito do conteúdo do enunciado. Foram obtidos escores individuais das crianças de ambos os grupos no pré e no pós-teste. Tais escores foram calculados somando-se algebricamente os pontos obtidos em cada um dos itens. Também foram calculados os valores mínimos e máximos, as medianas e os quartis de cada grupo no pré e no pós-teste.

Realizou-se uma análise estatística para a verificação da significância das diferenças entre os grupos. Primeiramente, foi aplicada a Prova de MannWhitney, comparando-se o pré-teste do grupo experimental com o pré-teste do grupo controle. Os resultados indicaram não haver diferença significativa entre os grupos no pré-teste, ou seja, estes puderam ser considerados equivalentes; pode-se afirmar que as amostras provêm de uma mesma população. Portanto, aplicou-se a Prova de Mann-Whitney comparando-se o pós-teste do grupo experimental com o pós-teste do grupo controle com o objetivo de verificar se a diferença entre os grupos era significativa no pós-teste, podendo se afirmar os efeitos da intervenção.

Também aplicou-se a Prova de Wilcoxon, comparando-se o pré-teste com o pós-teste do grupo experimental e o pré-teste com o pós-teste do grupo controle, para verificar em cada grupo, independentemente, se as diferenças entre os resultados do pré e do pós-teste eram significativas. 
Alguns dados das entrevistas foram cruzados com os dados da escala infantil, com o objetivo de verificar se há alguma relação entre os resultados obtidos na escala, referentes às atitudes em relação à inclusão, e os resultados obtidos a partir de algumas questões do roteiro de entrevistas.

Os dados das entrevistas abordados para o cruzamento com os dados da escala foram: 1) Contato prévio com pessoas com deficiência; 2) Acesso a informações sobre o tema; 3) Similaridades entre crianças com e sem deficiência; 4) Possibilidade de participação da criança deficiente nas atividades comuns à faixa etária; 5) Conceito de deficiência mental; 6) Escolarização na classe comum.

Para os itens 1 e 2 consideraram-se os dados dos grupos experimental e controle no pré-teste, por se referirem à caracterização inicial dos grupos. Para os itens 3, 5 e 6 consideraram-se apenas os dados do grupo experimental no pósteste, pois indicam os resultados do programa de intervenção. Para o item 4, consideraram-se os dados de ambos os grupo no pré e no pós-teste.

Cada grupo foi dividido em dois subgrupos, de acordo com os escores individuais obtidos na escala, sendo um subgrupo composto pelas crianças que obtiveram escores abaixo da mediana e o outro composto pelas crianças que obtiveram escores acima da mediana. Calculou-se para tais subgrupos a frequência com que cada categoria de respostas foi apontada em cada questão selecionada.

Compararam-se os dois subgrupos para cada questão, utilizando a Prova de Fisher. Assim, buscou-se verificar se o subgrupo de escores acima da mediana apresentou diferenças significativas do subgrupo abaixo da mediana com relação às questões selecionadas.

Para todos os cálculos estatísticos realizados nas diferentes etapas de análise dos dados, foram registrados os "valores de p". O nível de significância adotado em todos os testes foi de $\alpha=0,05$, ou seja, consideraram-se diferenças significativas aquelas cujo "valor de p" obtido foi menor que 0,05 .

Os testes foram aplicados de forma bicaudal, isto é, para verificar a significância, sem determinar o sentido da diferença. Nos casos em que as diferenças foram consideradas significativas, segundo esses cálculos, realizaramse os testes também de forma unicaudal, ou seja, indicando o sentido da diferença, de acordo com a hipótese inicial

\section{Resultados E discussões}

Inicialmente serão apresentados alguns dos resultados obtidos nas entrevistas com as crianças. Não serão apresentados de forma quantitativa, mas de um modo geral, para a contextualização e possibilidade de compreensão dos resultados apresentados posteriormente. Em seguida, serão apresentados os resultados da escala e posteriormente, resultados do cruzamento de dados da escala com dados das entrevistas. 


\subsection{Resultados Gerais dAS ENTREVISTAS}

A maioria das crianças disse ter contato com pessoas com deficiência. Não é possível afirmar que se referiram às pessoas com deficiência mental, pois as crianças evidenciaram desconhecimento sobre o tema. A escola é o local de maior contato, indicando que a presença de classes especiais, de salas de recursos e de alguns alunos com deficiência em salas comuns proporciona uma oportunidade para essa interação mista.

Muitas crianças afirmaram nunca terem tido acesso a informações sobre o tema. Entre as que tiveram, a televisão foi a fonte de informações mais citada, revelando o potencial da mídia para a construção de concepções mais adequadas e atitudes mais positivas quanto aos deficientes, devido ao grande alcance que possui. Os dados também enfatizam a necessidade de a mídia ampliar sua preocupação com os conteúdos veiculados, cuidando para não transmitir mensagens que mantenham estereótipos e preconceitos, nem que incentivem apenas a piedade e a caridade, no lugar da empatia e da solidariedade. Os resultados evidenciam a necessidade de pais e professores envolverem-se mais com o tema, procurando se informar sobre o assunto e rever suas próprias concepções, com o intuito de transformar o tema em algo natural, que faça parte do cotidiano escolar e familiar.

As crianças, de modo geral, não apresentaram atitudes preconceituosas ou de rejeição em relação às pessoas deficientes. Apesar disso, constatou-se um grande desconhecimento da deficiência mental, sendo esta confundida com outras deficiências, como a física e a sensorial, e relacionada a doenças. No último caso, várias crianças indicaram uma concepção do deficiente como frágil, não podendo se expor a situações sociais comuns, consideradas por tais crianças como fatores de risco à saúde dos deficientes.

Em sua maioria, as crianças pareceram perceber mais facilmente as deficiências que apresentam características de maior visibilidade, requerendo o uso de equipamentos ou evidenciando diferenças marcantes no corpo. Mesmo assim, já parecem perceber as distinções de pessoas com deficiência mental, apesar de terem dificuldade de compreendê-la, remetendo-se a comportamentos e traços da aparência que observam.

As crianças que passaram pelo programa apresentaram um maior entendimento de aspectos importantes da deficiência mental, como a dificuldade de aprendizado, o desenvolvimento mais lento e possíveis dificuldades de adaptações ao meio. O programa permitiu a compreensão das limitações da deficiência mental, diminuindo as confusões com outras deficiências e a relação com doenças. As crianças passaram a entender as necessidades dos deficientes mentais e a observar sua relação com o meio social.

O programa parece ter proporcionado a várias crianças, uma maior observação do meio e das dificuldades enfrentadas pelos deficientes, não apenas em virtude da deficiência, mas por sua relação com a sociedade, percebendo o tratamento recebido pelos deficientes e a necessidade de apoios e adaptações. 
O programa parece ter proporcionado, ainda, às crianças uma percepção das habilidades e possibilidades dos deficientes mentais e uma observação maior de similaridades entre eles e as pessoas sem deficiência, o que pode fazer com que os deficientes deixem de ser considerados estranhos e as crianças aumentem as iniciativas de aproximação a estes.

Quanto à educação formal dos deficientes, a maioria das crianças de ambos os grupos apresentou atitudes positivas e favoráveis, inclusive relacionadas à escolarização deles na escola comum. Com relação à escolarização na classe comum, houve divergência entre as crianças, inicialmente. As que passaram pelo programa apresentaram uma ampliação nas atitudes favoráveis à inclusão, diferindo significativamente do grupo controle, tanto nas entrevistas quanto na escala. Os relatos indicaram uma visão não apenas otimista, mas fundamentada na necessidade de apoio e nas adaptações na sala de aula, incluindo uma articulação entre o ensino comum e o ensino especial.

\subsection{RESUltados dA ESCALA INFANTIL DE ATITUDES SOCIAIS EM RELAÇÃO À INCLUSÃO}

Os resultados da aplicação da Escala infantil de atitudes sociais em relação à inclusão (BALEOTTI; OMOTE, 2003) são apresentados na Tabela 1, que indica os escores obtidos por cada participante, as medianas, os valores mínimos e máximos e os quartis.

Tabela 1 - Resultados da aplicação da Escala infantil de atitudes sociais em relação à inclusão

\begin{tabular}{ccccc}
\hline CRIANÇAS & \multicolumn{2}{c}{ GE - Escores } & \multicolumn{2}{c}{ GC- Escores } \\
\hline 1 & PRÉ & PÓS & PRÉ & PÓS \\
\hline 2 & -13 & 0 & -12 & -4 \\
3 & -8 & -2 & -10 & -10 \\
4 & 4 & 5 & -1 & -6 \\
5 & -11 & -5 & 1 & -1 \\
6 & -14 & 19 & -19 & -11 \\
7 & 0 & 7 & -6 & -9 \\
8 & 4 & -3 & -16 & -14 \\
9 & -3 & 2 & -5 & -6 \\
10 & -8 & -8 & -15 & -14 \\
11 & -11 & -2 & -18 & -14 \\
12 & -11 & 7 & -2 & -1 \\
\hline
\end{tabular}




\begin{tabular}{ccccc}
\hline CRIANÇAS & \multicolumn{2}{c}{ GE - Escores } & \multicolumn{2}{c}{ GC- Escores } \\
\hline 13 & PRÉ & PÓS & PRÉ & PÓS \\
14 & -15 & -12 & -5 & -8 \\
15 & 0 & 12 & -3 & 7 \\
16 & -11 & -8 & -1 & -3 \\
17 & 3 & 3 & -12 & -11 \\
18 & -7 & 2 & -8 & 0 \\
19 & 1 & 9 & 5 & -9 \\
20 & -8 & -2 & -17 & -10 \\
Mínimos & -9 & 5 & -8 & -15 \\
Quartis 1 & -15 & -12 & -19 & -15 \\
Medianas & -11 & $-3,25$ & $-15,25$ & $-11,5$ \\
Quartis 3 & -8 & 1 & -8 & -9 \\
Máximos & 0,25 & 5,5 & $-2,75$ & $-3,75$ \\
\hline
\end{tabular}

Pode-se observar que no grupo experimental os escores variaram de -15 a 9 no pré-teste, e de -12 a 19 no pós-teste, com as medianas -8 e 1, respectivamente. No grupo controle, os escores variaram de -19 a 5 no pré-teste, e de - 15 a 7 no pósteste, com as medianas -8 e -9 , respectivamente.

A síntese dos cálculos estatísticos realizados está apresentada na Tabela 2. Tabela 2 - Cálculos estatísticos da Escala infantil de atitudes sociais em relação à inclusão

\begin{tabular}{lcc}
\hline GRUPOS COMPARADOS & \multicolumn{2}{c}{ TESTES UTILIZADOS } \\
\hline GE no PRÉ com GC no PRÉ & $\mathrm{p}=0,2234$ & Wilcoxon \\
GE no PÓS com GC no PÓS & $\mathrm{p}=0,0004^{*}$ & - \\
GE no PRÉ com GE no PÓS & - & $\mathrm{p}=0,0047^{*}$ \\
GC no PRÉ com GC no PÓS & - & $\mathrm{p}=0,4900$ \\
\hline
\end{tabular}

* diferenças significativas; $\alpha=0,05$

GE no pré $=$ GC no pré

GE no pós ₹ GC no pós; GE > GC ( $p=0,0002)$

GE no pré $\neq$ GE no pós; GE no pós $>$ GE no pré $(p=0,0024)$

GC no pré $=$ GC no pós 
É possível perceber que a intervenção trouxe implicações para as atitudes sociais das crianças do estudo em relação à inclusão. Os resultados obtidos com o Teste de Mann-Whitney indicam que os grupos não diferiam significativamente no pré-teste quanto às atitudes sociais em relação à inclusão $(p=0,2234)$. Logo, pode-se afirmar que os grupos eram equivalentes no pré-teste, ou seja, provinham de uma mesma população. Após a intervenção, isso se modifica, isto é, há uma diferença significativa entre os grupos, tendo o GE apresentado maior favorabilidade à inclusão do que o GC ( $\mathrm{p}=0,0004)$.

A aplicação do teste de Wilcoxon confirma esses achados. Comparandose o pré-teste do GE com o pós-teste do mesmo, evidencia-se uma diferença significativa, ou seja, as crianças apresentaram atitudes mais favoráveis à inclusão após passarem pelo programa informativo $(p=0,0047)$. Já no $G C$, não há diferença significativa entre os dados do pré e do pós-testes $(p=0,49)$. Pode-se perceber que enquanto as crianças do grupo controle não apresentaram mudanças significativas em suas atitudes sociais quanto à inclusão, as crianças do grupo experimental apresentaram mudanças significativas. Como os grupos eram equivalentes no pré-teste, considera-se que as mudanças nessas atitudes foram provenientes da intervenção. Assim, os resultados obtidos com a escala indicam que o programa informativo foi capaz de transformar as atitudes sociais das crianças em relação à inclusão, aumentando as atitudes positivas, isto é, as atitudes favoráveis a esta.

O conteúdo trabalhado no programa informativo sobre a deficiência mental incluiu a temática da escolarização dos deficientes e algumas modalidades de ensino possíveis, indicando seus objetivos, sua atuação, suas dificuldades e suas vantagens. Tais conhecimentos, aliados aos esclarecimentos das reais limitações das pessoas com deficiência mental, bem como de suas habilidades e possibilidades, devem ter contribuído para as mudanças nas atitudes das crianças quanto à inclusão. Uma vez que a escala não se refere apenas à deficiência mental, pode-se afirmar que a mudança nas atitudes das crianças indica uma maior aceitação dos alunos com diversas deficiências nas salas regulares, um interesse na interação social e uma maior proximidade das crianças com deficiência.

Os resultados obtidos com a aplicação da escala parecem estar em consonância com os dados obtidos nas entrevistas, nas quais não foram evidenciadas diferenças entre os grupos, em relação à escolarização das crianças com deficiência na escola regular, uma vez que já no pré-teste a maioria das crianças mostrou-se favorável a isso. Todavia, com relação à escolarização em salas regulares, houve diferença significativa entre os grupos no pós-teste, tendo o grupo experimental apresentado mais respostas favoráveis à inclusão de crianças deficientes nas salas comuns do que o grupo controle.

A confirmação dos efeitos do programa informativo sobre as atitudes relacionadas à inclusão de deficientes em salas regulares amplia a abrangência do programa informativo original, de Ferreira (1998). Tais resultados indicam o valor desse programa informativo no processo de inclusão. Segundo Stainback 
e Stainback (1999), para que a inclusão ocorra de fato é necessário que a escola desenvolva uma cultura da diversidade. Vários estudos, como os de Guralnick et al. (1995); Jacques et al. (1998); e York et al. (1992), apontam as possibilidades de benefícios tanto para os deficientes como para as crianças sem deficiência nesse processo, no sentido de desenvolverem tolerância, respeito mútuo, cooperação. Logo, ressalta-se a necessidade de as escolas e as comunidades que se propõem à inclusão desenvolverem estratégias informativas e de sensibilização referentes às diferenças e, especificamente, às deficiências, desde a infância, com o intuito de ampliar as atitudes de solidariedade e a valorização das diferenças.

\subsection{Cruzando dados das ENTREVISTAS COM DAdOS DA ESCALA INFANTIL DE ATI- TUDES SOCIAIS EM RELAÇÃO À INCLUSÃO}

Atitudes sociais em relação à inclusão $X$ Contato prévio com pessoas com deficiência e Atitudes sociais em relação à inclusão $X$ Acesso a informações sobre o tema.

Para o cruzamento destes dados, consideraram-se, separadamente, os resultados do grupo experimental e os do grupo controle, no pré-teste.

Estudiosos na área da educação especial, como Werneck (1997) e Ferreira (1998), por exemplo, indicam a importância do contato e da informação para a construção das atitudes sociais em relação às pessoas deficientes, revelando atitudes mais positivas quando o contato com pessoas deficientes e o acesso a informações são maiores. A hipótese quanto a este cruzamento era que o subgrupo acima da mediana, ou seja, de crianças que indicaram na escala atitudes mais favoráveis à inclusão, seria composto por crianças que tinham mais contato com pessoas com deficiência e mais acesso a informações sobre o tema, comparandose com o subgrupo de crianças abaixo da mediana. No entanto, tratando-se do contato prévio com pessoas com deficiência e do acesso a informações, tanto no GC quanto no GE constatou-se não haver diferença estatisticamente significativa entre os subgrupos de crianças acima e o abaixo da mediana, isto é, indicam que as crianças que apresentaram atitudes mais favoráveis à inclusão não diferiram das que apresentaram atitudes menos favoráveis, em relação ao contato prévio com pessoas com deficiência ou o acesso a informações sobre o tema por intermédio da televisão, dos pais ou dos professores. As frequências para essas respostas são apresentadas nas tabelas a seguir: 
Tabela 3 - Cruzamento de dados da escala com respostas sobre o contato prévio com deficientes - grupo experimental/pré-teste

\begin{tabular}{ccc}
\hline & Grupo acima da mediana & Grupo abaixo da mediana \\
\hline SIM & 7 & 6 \\
NÃO & 3 & 4 \\
\hline TOTAL & 10 & 10 \\
\hline
\end{tabular}

Questão norteadora: Você já tinha visto/conhecido pessoas ou crianças com deficiência mental?

Tabela 4 - Cruzamento de dados da escala com respostas sobre o contato prévio com deficientes - grupo controle/pré-teste

\begin{tabular}{ccc} 
& Grupo acima da mediana & Grupo abaixo da mediana \\
\hline SIM & 8 & 9 \\
NÃO & 2 & 1 \\
\hline TOTAL & 10 & 10
\end{tabular}

Questão norteadora: Você já tinha visto/conhecido pessoas ou crianças com deficiência mental?

Tabela 5 - Cruzamento de dados da escala com respostas sobre acesso a informações sobre o tema - grupo experimental/pré-teste

\begin{tabular}{ccc}
\hline & Grupo acima da mediana & Grupo abaixo da mediana \\
\hline SIM & 5 & 4 \\
NÃO & 5 & 6 \\
\hline TOTAL & 10 & 10
\end{tabular}

Questão norteadora: Você já tinha ouvido falar/alguém já te explicou algo sobre as crianças com deficiência mental? 
Tabela 6 - Cruzamento de dados da escala com respostas sobre acesso a informações sobre o tema - grupo controle/pré-teste

\begin{tabular}{ccc} 
& Grupo acima da mediana & Grupo abaixo da mediana \\
\hline SIM & 6 & 3 \\
NÃO & 4 & 7 \\
\hline TOTAL & 10 & 10 \\
\hline
\end{tabular}

Questão norteadora: Você já tinha ouvido falar/alguém já te explicou algo sobre as crianças com deficiência mental?

Os valores encontrados no Teste de Fisher foram $p=1$, para ambos os grupos, referente ao contato prévio; $\mathrm{p}=1$, no GE, e p = 0,3698, no GC, relacionados ao acesso a informações sobre o tema.

É possível levantar a hipótese de que mesmo as crianças que disseram ter contato com pessoas deficientes ou acesso a informações sobre o tema não tiveram suas atitudes sociais em relação à inclusão, influenciadas por estas. Isso pode ter ocorrido pelo fato de tais informações e contatos estarem sendo insuficientes ou por não abordarem diretamente a temática da inclusão, ou, ainda, por não afetarem de modo significativo as crianças em suas atitudes quanto ao tema. Esses resultados enfatizam a necessidade de os meios de comunicação estarem atentos às mensagens veiculadas sobre as pessoas deficientes, bem como os pais e os educadores em relação às informações a que as crianças têm acesso e às interações que estabelecem, que devem ocorrer de modo a ressaltar as possibilidades dos deficientes, além de suas necessidades, e de indicar os benefícios desse contato para todos os envolvidos.

Atitudes sociais em relação à inclusão $X$ Similaridades entre pessoas com e sem deficiência e Atitudes sociais em relação à inclusão X Participação nas atividades comuns à faixa etária.

Para esses cruzamentos, considerou-se o grupo experimental, no pósteste. As hipóteses eram de que o subgrupo acima da mediana apresentaria mais respostas "sim" do que "não", comparando-se com o subgrupo abaixo da mediana, quanto às similaridades entre crianças com e sem deficiência e à possibilidade de participação dos deficientes nas atividades comuns à faixa etária.

De fato, a frequência de respostas "sim" para ambas perguntas foi maior no subgrupo acima da mediana do que as respostas "não", enquanto no grupo abaixo da mediana, a frequência de respostas "não" foi maior ou a mesma, indicando que as crianças com atitudes mais positivas em relação à inclusão também revelaram maior percepção de similaridades entre deficientes e não-deficientes, bem como 
das possibilidades de participação dos deficientes nas atividades comuns à faixa etária. As frequências para essas respostas são apresentadas nas tabelas a seguir:

Tabela 7 - Cruzamento de dados da escala com respostas sobre a similaridade entre pessoas com e sem deficiência - grupo experimental/pós-teste

\begin{tabular}{ccc} 
& Grupo acima da mediana & Grupo abaixo da mediana \\
\hline SIM & 6 & 3 \\
NÃO & 4 & 7 \\
\hline TOTAL & 10 & 10 \\
\hline
\end{tabular}

Questão norteadora: “A criança com deficiência mental é igual a você e as outras crianças?"

Tabela 8 - Cruzamento de dados da escala com respostas sobre a participação de pessoas com deficiência nas atividades comuns à faixa etária - grupo experimental/pós-teste

\begin{tabular}{ccc} 
& Grupo acima da mediana & Grupo abaixo da mediana \\
\hline SIM & 8 & 5 \\
NÃO & 2 & 5 \\
\hline TOTAL & 10 & 10 \\
\hline
\end{tabular}

Questão norteadora: As crianças com deficiência mental podem fazer tudo o que você faz?

Apesar das diferenças observadas entre os grupos, essas não foram consideradas estatisticamente significativas. Os valores encontrados no Teste de Fisher foram: $p=0,3698$, referente às similaridades, e $p=0,3498$, com relação à participação nas atividades. Logo, não é possível afirmar que as crianças que apresentaram atitudes mais favoráveis à inclusão foram as que visualizaram mais similaridades entre pessoas com e sem deficiência e mais habilidades dos deficientes.

\section{Atitudes sociais em relação à inclusão $X$ Conceito de deficiência mental}

Para esse cruzamento, considerou-se o grupo controle e o grupo experimental no pré e no pós-teste, porque quase todas as crianças do GE, no pós-teste, deram respostas referentes à mesma categoria. Logo, comparar apenas os subgrupos do experimental, nesse caso, não permitiria uma afirmação correta sobre tal cruzamento.

A hipótese era que as crianças com atitudes mais favoráveis quanto à inclusão poderiam apresentar concepções mais adequadas sobre a deficiência 
mental, porém não foram encontradas diferenças significativas entre os subgrupos abaixo e acima da mediana referentes aos conceitos de deficiência mental apresentados - no caso, comparando-se as categorias de respostas relacionadas à deficiência física e sensorial e as associadas às características da própria deficiência mental. Assim, as crianças que indicaram atitudes mais favoráveis à inclusão não foram, necessariamente, as que apresentaram concepções mais adequadas da deficiência mental.

As frequências para essas respostas são apresentadas nas tabelas a seguir:

Tabela 9 - Cruzamento de dados da escala com respostas sobre o conceito de deficiência mental - grupo experimental/pré-teste

Grupo acima da mediana Grupo abaixo da mediana

\begin{tabular}{ccc}
\hline Deficiência mental & 2 & 1 \\
Def. física/sensorial & 8 & 6 \\
\hline TOTAL & 10 & 7 \\
\hline
\end{tabular}

Questão norteadora: O que é uma criança “deficiente mental”?

Tabela 10 - Cruzamento de dados da escala com respostas sobre o conceito de deficiência mental - grupo experimental/pós-teste

\begin{tabular}{ccc}
\hline & Grupo acima da mediana & Grupo abaixo da mediana \\
\hline Deficiência mental & 9 & 9 \\
Def. física/sensorial & 1 & 1 \\
\hline TOTAL & 10 & 10 \\
\hline
\end{tabular}

Questão norteadora: O que é uma criança “deficiente mental”?

Tabela 11 - Cruzamento de dados da escala com respostas sobre o conceito de deficiência mental - grupo controle/pré-teste

\begin{tabular}{ccc}
\hline & Grupo acima da mediana & Grupo abaixo da mediana \\
\hline Deficiência mental & 0 & 3 \\
Def. física/sensorial & 6 & 6 \\
\hline TOTAL & 6 & 9 \\
\hline
\end{tabular}

Questão norteadora: O que é uma criança "deficiente mental"? 
Tabela 12 - Cruzamento de dados da escala com respostas sobre o conceito de deficiência mental - grupo controle/pós-teste

\begin{tabular}{ccc}
\hline & Grupo acima da mediana & Grupo abaixo da mediana \\
\hline Deficiência mental & 3 & 4 \\
Def. física/sensorial & 6 & 6 \\
\hline TOTAL & 9 & 10 \\
\hline
\end{tabular}

Questão norteadora: O que é uma criança "deficiente mental”?

Os valores encontrados no Teste de Fisher foram $p=1$, no GE e $p=0,2286$, no GC, no pré-teste; e $\mathrm{p}=1,5263$, no GE, e $\mathrm{p}=1$, no GC, no pós-teste.

Atitudes sociais em relação à inclusão X Escolarização de deficientes na classe comum.

Para tal cruzamento, consideraram-se os dados do grupo experimental, no pós-teste. Tanto essa questão quanto a escala se propõem a investigar o mesmo fenômeno, ou seja, as atitudes em relação à inclusão de deficientes em salas regulares. A hipótese era que o subgrupo acima da mediana apresentaria mais respostas positivas ("sim") do que respostas negativas ("não") quando questionado sobre a escolarização de deficientes em classes comuns, comparando-o com o subgrupo abaixo da mediana. As frequências para essas respostas são apresentadas na tabela a seguir:

Tabela 13 - Cruzamento de dados da escala com respostas sobre a escolarização de deficientes no ensino comum - grupo experimental/pós-teste

\begin{tabular}{ccc}
\hline & Grupo acima da mediana & Grupo abaixo da mediana \\
\hline SIM & 10 & 4 \\
NÃO & 0 & 6 \\
\hline TOTAL & 10 & 10 \\
\hline
\end{tabular}

Questão norteadora: As crianças com deficiência mental podem estudar na sua classe?

Verificou-se uma diferença estatisticamente significante entre os subgrupos, confirmando a hipótese anterior - as crianças que tiveram maior escore na escala deram mais respostas positivas que negativas em relação à escolarização de deficientes em classes comuns, quando comparadas às crianças com menores escores na escala $(p=0,0108)$. Pode-se afirmar, portanto, que a escala utilizada confirmou-se como um instrumento eficaz para medir as atitudes sociais referentes à inclusão de alunos deficientes nas salas regulares. 


\section{Conclusões}

Como se observa nas Tabelas 1 e 2, o programa informativo desenvolvido ampliou as atitudes favoráveis à inclusão; porém, como indicam os resultados imediatamente descritos, no presente estudo, não se pôde afirmar estatisticamente que as crianças que apresentaram atitudes mais favoráveis relacionadas à inclusão foram as que tinham mais contato e acesso a informações prévias sobre o tema, nem que estas foram as que indicaram concepções mais adequadas da deficiência mental. Observou-se uma tendência à percepção de mais similaridade entre pessoas com e sem deficiência e das possibilidades de participação dos deficientes nas atividades comuns à faixa etária, entre as crianças mais favoráveis à inclusão, comparando-se com as crianças menos favoráveis, no entanto também não se pôde afirmar isso estatisticamente. Não é possível afirmar exatamente quais aspectos trabalhados na intervenção promoveram tal efeito. Os dados indicam que a articulação das variadas informações sobre o tema deve ter promovido as mudanças nas atitudes das crianças, e não conteúdos específicos isoladamente.

Tendo em vista esses resultados, permanece a necessidade de ampliação das pesquisas sobre as relações entre os fenômenos apresentados. Talvez sejam necessários estudos que envolvam: a observação direta dos contatos entre crianças com e sem deficiência; o acesso às informações prévias recebidas pelas crianças por meio dos programas televisivos; entrevistas com os pais sobre o tema; estudos mais amplos, com instrumentos variados sobre a percepção de similaridades e habilidades dos deficientes; a aceitação do mesmo e as atitudes sociais das crianças em situações concretas de interação social e inclusão, e não apenas hipotéticas.

Pode-se afirmar, de modo geral, que o programa proporcionou um maior conhecimento sobre a deficiência mental, incluindo uma visão mais realista das possíveis limitações, das habilidades de pessoas deficientes e das necessidades de apoio e transformações do meio para atender às necessidades delas. Além disso, aumentou a percepção das similaridades entre pessoas deficientes e nãodeficientes, a aceitação social do deficiente entre as crianças, a disponibilidade em ajudar e especialmente, as atitudes positivas em relação à inclusão.

Entende-se que a proposta da inclusão envolve uma série de mudanças de ordem política, estrutural e pedagógica. É essencial, porém, que ocorram também mudanças de ordem social e afetiva, pois a inclusão envolve pessoas em interação, indivíduos com valores, crenças e reações emocionais. Portanto, a inclusão deve ser entendida também em seu aspecto relacional, devendo ser ampliadas pesquisas e intervenções acerca das concepções, dos sentimentos e das atitudes das pessoas sem deficiência em relação aos deficientes e à inclusão, uma vez que eles provavelmente vão influenciar as interações sociais estabelecidas. 


\section{REFERENCIAS}

AMARAL, L. A. O espelho convexo: o corpo desviante no imaginário coletivo pela voz da literatura infanto-juvenil. 1992. 399f. Tese (Doutorado em Psicologia) - Instituto de Psicologia, Universidade de São Paulo, São Paulo, 1992.

BALEOTTI, L. R.; OMOTE, S. Atitudes sociais de alunos do ciclo I do ensino fundamental em relação à inclusão: construção de uma escala infantil. In: SIMPÓSIO EM FILOSOFIA E CIÊNCIAS, 5., 2003. Anais... Marília: Universidade Estadual Paulista, 2003.

BEM, D. J. Convicções, atitudes e assuntos humanos. São Paulo: Edusp, 1973.

DENARI, F. E. Universo e destinação de adultos com deficiência mental: respostas institucionais a seus direitos de cidadania. In: MARTINS, L. A. R. et al. (Org.). Inclusão: compartilhando saberes. Petrópolis: Vozes, 2006. cap.2, p.206-323.

FERREIRA, S. L. Aprendendo sobre a deficiência mental: um programa para crianças. São Paulo: Memnon, 1998.

GURALNICK, M. J. et al. Immediate effects of mainstreamed settings on the social interactions and social integration of preschool children. American Journal of Mental Retardation: AJMR, Washington, v.100, n.4, p.359-377, 1995.

JACQUES, N.; WILTON, K.; TOWNSEND, M. Cooperative learning and social acceptance of children with mild intellectual disability. Journal of Intellectual Disability Research, Oxford, v.42, n.1, p.29-36, 1998.

MARTINS, G. A. H. A integração do aluno deficiente na classe comum: o ponto de vista de alunos do ciclo I do ensino fundamental. 1999. 206f. Dissertação (Mestrado em Educação) - Programa de Pós-graduação em Educação, Faculdade de Filosofia e Ciências, Universidade Estadual Paulista, Marília, 1999.

OMOTE, S. Deficiência e não-deficiência: recortes do mesmo tecido. Revista Brasileira de Educação Especial, Marília, v.1, n.2, p.65-73, 1994.

- A construção de uma escala de atitudes sociais em relação à Inclusão: notas preliminares. Revista Brasileira de Educação Especial, Marília, v.11, n.1, p.33-48, 2005.

STAINBACK, S.; STAINBACK, W. Inclusão: um guia para educadores. Porto Alegre: Artmed, 1999.

VIEIRA, C. M. Programa informativo sobre deficiência mental e inclusão: efeitos nas atitudes e concepções de crianças não-deficientes. 2006. 208f. Dissertação (Mestrado em Educação Especial) - Programa de Pós-graduação em Educação Especial, Universidade Federal de São Carlos, São Carlos, 2006.

WERNECK, C. Ninguém mais vai ser bonzinho, na sociedade inclusiva. Rio de Janeiro: WVA, 1997.

YORK, J. et al. Feedback about integrating middle-school students with severe disabilities in general education classes. Exceptional Children, Reston, v.58, n.3, p.244-258, 1992.

Recebido em: 01/07/2011

Reformulado em: 18/01/2012

Aprovado em: 30/03/2012 